\title{
INDIVIDUACIÓN Y LENGUAJE NO VERBAL EN LA CMC
}

\section{INDIVIDUATION AND NON-VERBAL LANGUAGE IN CMC}

\section{Anthony López Get*}

\begin{abstract}
RESUMEN
Este artículo analiza el uso de claves no verbales - tales como los emoticones, las claves tipográficas, entre otros - en la comunicación mediada por computadora (CMC). Se pretende demostrar cómo estas claves reproducen elementos kinésicos y paralingüísticos de la comunicación cara a cara que son esenciales para lograr transmitir, de forma más efectiva, un mensaje — factor clave para la socialización y la individuación - para así superar, aunque sea parcialmente, la barrera comunicativa que presenta la comunicación escrita despersonalizada de los medios electrónicos.
\end{abstract}

PALABRAS CLAVE: COMUNICACIÓN MEDIADA POR COMPUTADORA * CLAVES NO VERBALES * INDIVIDUACIÓN * SOCIALIZACIÓN * SUBJETIVIDAD

\section{ABSTRACT}

This article analyzes the use of non-verbal cues — such as emoticons, typographical clues, among others - in computer-mediated communication (CMC). It intends to demonstrate how these cues reproduce kinesic and paralinguistic elements of the face-to-face communication which are essential in transmitting a message in an effective way - a key factor to the socialization and individuation - in order to overcome, even partially, the communicative barrier of the depersonalized written communication of the electronic media.

KEYWORDS: COMPUTER MEDIATED COMMUNICATION * NON-VERBAL CUES * INDIVIDUATION * SOCIALIZATION * SUBJECTIVITY

Escuela de Lenguas Modernas, Universidad de Costa Rica.

tonyget@gmail.com 
La comunicación entre seres humanos es parte esencial del desarrollo del individuo en sociedad. Tal y como lo expone Habermas, en su estudio "Individuación por vía de socialización: sobre la teoría de la subjetividad de George Herbert Mead" (1990), el sujeto determina su individualidad por medio de la socialización, la cual es mediada por el lenguaje y propone que:

...el significado de la expresión "individualidad" hay que explicarlo por referencia a la autocomprensión de un sujeto capaz de lenguaje y de acción, que se presenta $y$, llegado el caso, se justifican ante los demás participantes en la conversación como una persona incanjeable e inconfundible. Esta autocomprensión, por difusa que pueda ser, funda la identidad del yo (p. 207).

De forma similar, Michael Tomasello en Origins of human communication (2008), señala que "el compartir con otros de esta forma sirve para expandir la propia base común [common ground] — ser como otros en el grupo y, uno espera, ser aceptado por ellos y ser capaz de comunicarse con ellos más íntimamente- $y$ por ende sirve como forma de identificación social y de vinculación [bonding]" (traducción propia) ${ }^{1}$ (p.282). Es decir, es en el lenguaje donde el sujeto logra su individuación al ser esta validada por otros individuos del grupo social, de ahí la importancia de que la comunicación sea efectiva, en términos de las demandas del grupo social en el que el sujeto se desenvuelve.

Hoy en día, la comunicación mediada por computadora $(\mathrm{CMC})$ representa uno de los medios de interacción entre individuos más populares dadas sus características de rapidez, relativo bajo costo - si se cuenta con el equipo necesario-y accesibilidad.

$1 \quad$ Sharing with others [...] serves to expand one's common ground with others - to be like others in the group and to, one hopes, be liked by them and be able to communicate with them more intimately - and so serves as a form of social identification and bonding (Tomasello, 2008: 282).
No solo el correo electrónico, sino también los chatrooms y aplicaciones de mensajería instantánea, tales como: Messenger (MSN), Google Talk, entre muchos otros, los chats de los llamados videojuegos de rol multijugador masivo en línea (MMORPG por sus siglas en inglés) y los mensajes de texto por teléfono móvil, han cambiado la forma en que las personas se comunican y utilizan el lenguaje ${ }^{2}$. Sin embargo, la efectividad de la comunicación por estos medios electrónicos - por ejemplo, en cuanto a la constante presencia de malas interpretaciones del mensaje o a la supuesta imposibilidad de una individuación de los interlocutores - ha sido debatida por algunas $y$ algunos investigadores, en tanto carecen de las características claves no verbales que el ser humano utiliza en la comunicación cara a cara, claves que facilitan tanto la comprensión del mensaje como el reconocimiento de los interlocutores como sujetos.

Según Duke, un 65\% de la comunicación social es no verbal (1974: 397), lo que indica la importancia que tienen estas señales en la interacción comunicativa. En "The nonverbal communication functions of emoticons in computer-mediated communication", ShaoKang Lo (2008), expone los problemas que la falta de claves no verbales genera en la CMC:

Otra discusión importante acerca de la ausencia de información del contexto social es el enfoque de claves filtradas, el cual sostiene que la CMC carece de claves no verbales para transmitir conversaciones socialmente orientadas, causando así una mala interpretación por parte

2 Todas estas formas de comunicación electrónica, a pesar de las características propias de cada medio, comparten la forma básica de comunicación textual (chats, mensajes de texto) y por ende, comparten también las estrategias utilizadas por los y las usuarias para solventar las limitaciones comunicativas que poseen. Para los efectos de este estudio, tomaremos en cuenta medios de CMC que utilizan mensaje textual (texto escrito), con algunas variantes $y$ excluiremos los medios que cuentan con modo de video llamada, en tanto son menos utilizados y poseen características y limitaciones propias. 
del receptor de la verdadera intención del remitente y que da como resultado conflictos (traducción propia) ${ }^{3}$ (p. 595).

Las claves no verbales ayudan a los interlocutores a interpretar el mensaje de acuerdo con las señales - expresiones faciales, proximidad, tono de voz, entre otras- que acompañan al mensaje verbal, a generar una idea, una impresión del "otro" en el lenguaje en términos de sus emociones y su actitud. Al estar dichas señales ausentes en la CMC, el mensaje puede ser malinterpretado - tal y como lo expone Lo- $y$ el reconocimiento del sujeto por parte de los otros se ve limitado al texto escrito despersonalizado.

No obstante, si bien los medios electrónicos de comunicación carecen de un acceso directo a las claves no verbales de la comunicación cara a cara, los usuarios de este tipo de herramientas han desarrollado una serie de estrategias para substituir dicha carencia, al menos, de forma parcial y así lograr una comunicación más efectiva, facilitando la creación de relaciones interpersonales entre interlocutores, incluso cuando nunca se han visto las caras. El uso de claves similares a las claves no-verbales (o cuasi-no verbales) en la $\mathrm{CMC}$, expone la necesidad en los seres humanos de recurrir a este tipo de señales para lograr una comunicación efectiva en la que los gestos, la pantomima, el lenguaje corporal y otras claves no verbales semejantes - que según Tomasello (2008) son la base del origen de la comunicación humana- logran llenar, con ciertas limitaciones, claro está, los vacíos que deja la simple escritura despersonalizada $y$ le permiten a los individuos revelarse e identificarse entre sí como sujetos reales en un medio, considerado con anterioridad, comunicativamente estéril e impersonal.

$3 \quad$ Another important discussion about the absence of social context information is the cues-filteredout approach, which holds that CMC lacks nonverbal cues to convey socially oriented conversations causing receivers to misunderstand the actual intent of the sender and resulting in conflicts (Lo, 2008: 595).

\section{LAS CLAVES NO VERBALES: ORIGEN Y FUNCIÓN}

Las claves no verbales son vitales en la comunicación humana. No solo acompañan al lenguaje verbal en la comunicación cara a cara para hacerla más efectiva, sino que logran transmitir un mensaje sin necesidad de una explicación verbal. Tomasello señala la importancia de estas formas no verbales de comunicación, al proponer que la comunicación humana convencional evolucionó a partir de un lenguaje "natural", basado en expresiones no verbales, como lo son el señalar (pointing) $y$ la pantomima (pantomiming), que representan un punto de transición en la estructuración del lenguaje convencional oral (2008: 2) y también dice:

Si por lo tanto queremos comprender la comunicación humana, no podemos empezar con el lenguaje. Debemos más bien comenzar con la comunicación no convencionalizada o codificada $y$ otras formas de sintonía mental como base. Los gestos humanos naturales, tales como el señalar y la pantomima, son excelentes candidatos para este rol (traducción propia) ${ }^{4}$ (p.59).

En su estudio, Tomasello compara el uso de claves no verbales en humanos y en primates no humanos para fundamentar su teoría y concluye que, lo que nos diferencia de los primates no humanos en el proceso evolutivo del lenguaje es la naturaleza cooperativa de la comunicación humana: "La comunicación humana es por tanto, una empresa fundamentalmente cooperativa, que opera más natural y fluidamente dentro del contexto de (1) una base conceptual común mutualmente asumida y (2) de motivos comunicativos cooperativos mutualmente asumidos" (traducción

4 If we want to understand human communication, therefore, we cannot begin with language. Rather, we must begin with unconventionalized, uncoded communication, and other forms of mental attunement, as foundational. Excellent candidates for this role are humans' natural gestures such as pointing and pantomiming (Tomasello, 2008: 59). 
propia) $)^{5}$ (2008: 6). Gracias a esta "intencionalidad compartida" (shared intentionality) y a la "base conceptual común" (common conceptual ground) es que los seres humanos fueron capaces de evolucionar comunicativamente, de generar los diferentes lenguajes convencionales que se utilizan hoy $y$ de posicionarse como individuos en el contexto social. Sin embargo, según Tomasello, es a partir del señalar y de la pantomima, que la comunicación humana se originó: "Específicamente, el señalar se basa en la tendencia natural de los humanos a seguir la dirección de la mirada de otros hacia blancos externos y la pantomima se basa en la tendencia natural de los humanos a interpretar intencionalmente las acciones de otros" (traducción propia) ${ }^{6}$ (2008: 9). Este autor contrasta la comunicación convencional más artificial si se quiere, a la comunicación no verbal, por medio del señalar y la pantomima, de carácter "natural" y enfatiza el hecho de que tanto el señalar, como los gestos icónicos no requieren de una base lingüística, al comprobar su uso en niños en etapas tempranas prelingüísticas (2008: 70).

Ahora bien, las claves no verbales no se limitan a señalar o a hacer pantomima. Existe toda una variedad de claves no verbales que, en conjunto, conforman lo que llamamos comunicación no verbal. Hecht; DeVito y Guerrero definen la comunicación no verbal como "todos los mensajes que no sean palabras que las personas intercambian en contextos interactivos" (traducción propia) ${ }^{7}$ (citados en Hein y Turkstra, 2003). En "Show me what you mean: nonverbal communication theory and application", Hein

$5 \quad$ Human communication is thus a fundamentally cooperative enterprise, operating most naturally and smoothly within the context of (1) mutually assumed common conceptual ground, and (2) mutually assumed cooperative communicative motives (Tomasello, 2008: 6).

$6 \quad$ Specifically, pointing is based on humans' natural tendency to follow the gaze direction of others to external targets, and pantomiming is based on humans' natural tendency to interpret the actions of others intentionally (Tomasello, 2008: 9).

All the messages other than words that people exchange in interactive contexts (citados en Hein $y$ Turkstra, 2003). y Turkstra proponen que el comportamiento humano tiene el potencial de ser comunicativo en tanto sea intencional y sea comprendido como mensaje por una comunidad de hablantes: "La comunicación no verbal incluye aquellos comportamientos que son reconocidos mutuamente $y$ los códigos y patrones compartidos socialmente con un enfoque en el significado del mensaje" (traducción propia) ${ }^{8}$ (2003). La comunicación no verbal, explican Hein y Turkstra, cumple las funciones de crear una impresión ante los actores, controlar la interacción, expresar emociones, identificar el engaño, entre otras $y$ categorizan el comportamiento no verbal de la siguiente forma:

$\diamond \quad$ Kinésicos: mensajes enviados por el cuerpo, incluyendo gestos, expresiones faciales, movimientos corporales, postura, mirada y forma de andar.

$\diamond \quad$ Vocálicos (e.g. paralingüístico): claves vocales diferentes a las palabras, incluyendo volumen, ritmo, tono, pausas y silencio.

$\diamond \quad$ Apariencia física: claves manipulables relacionadas con el cuerpo, incluyendo corte de cabello, vestimenta, cosméticos $y$ fragancia.

$\diamond \quad$ Táctil: claves de contacto, tales como frecuencia, intensidad y tipo de toque.

$\diamond \quad$ Proxemia: claves espaciales, incluyendo distancia interpersonal, territorialidad $y$ otras relaciones espaciales.

$\diamond \quad$ Cronemia: el uso del tiempo como sistema de mensaje, incluyendo puntualidad, cantidad de tiempo que se pasa con algún otro y tiempo de espera.

$\diamond \quad$ Artefactos: objetos manipulables del ambiente que pueden reflejar mensajes del diseñador o del usuario, tales como muebles, arte, mascotas $u$ otras posesiones (2003, traducción propia).

Como se puede ver, estas categorías están ausentes o se ven altamente limitadas en

$8 \quad$ Nonverbal communication includes those behaviors that are mutually recognized and socially shared codes and patterns with a focus on message meaning (Hein y Turkstra, 2003). 
la mayoría de formas de CMC, al encontrarse los interlocutores usualmente separados espacialmente ${ }^{9}$. Ahora, como lo mencionamos anteriormente, este distanciamiento y la falta de claves no verbales ha sido substituida por lo que tanto Lo (2008) como Carter (2003), llaman "claves cuasi-no verbales", entre las que incluiremos el uso de emoticones -tipográficos y animados-, claves tipográficas - como el uso de mayúsculas para indicar un cambio en el tono de "voz" - y lo que se llamará "comandos gesticulares" o emotes, entre otras. La efectividad de este tipo de claves cuasi-no verbales - principalmente en los emoticones, sobre los cuales se han realizado más estudios- ha sido cuestionada por los que afirman, como lo hace Carter que "no existe substituto para la interacción no verbal cara a cara" (traducción propia) ${ }^{10}$ (2003: 31), mientras que otros aseguran que, aunque con limitaciones, estas técnicas vienen a compensar de forma satisfactoria la ausencia de una lectura real de claves no verbales similar a la que se da en la comunicación cara a cara.

\section{LA AUSENCIA DE CLAVES NO VERBALES EN LA CMC}

En "Exploring the cognitive-emotional fugue in electronic negotiations", Griessmair $y$ Koeszegi (2009) citan a von Thun, quien propone que:

Un mensaje contiene no solo un único significado sino que consta de cuatro distintas capas comunicativas. La primera comprende el contenido factual del mensaje, por ejemplo, acerca de los que uno informa a su contraparte. La segunda, auto revelación, se refiere a lo que uno revela sobre uno mismo por medio del

$9 \quad$ Incluso al encontrarse los interlocutores en el mismo espacio - en cubículos contiguos, por ejemplo-, si la comunicación se da por medios electrónicos, la función de las categorías antes mencionadas se anula o se limita, al no estar los sujetos, literalmente, cara a cara.

10 No substitutes exist for nonverbal, face-to-face interaction (Carter, 2003: 31). mensaje. La tercera provee información acerca de las relaciones entre los comunicadores, por ejemplo, lo que uno piensa acerca del otro y cómo el primero se relaciona con el o la última. La cuarta, atracción, indica a lo que uno quiere inducir a su contraparte (traducción propia) ${ }^{11}$ (p. 216-217).

En la CMC, según sus críticos, solo la primera fase se puede dar efectivamente, ya que las otras tres dependen mucho de la presencia del individuo. Derks; Fischer y Bos (2008) mencionan que:

Las muestras no verbales tienen muchas funciones sociales. Una función es la reducción en la ambigüedad de la expresión intencional de emociones. Por ejemplo, un mensaje podría tener un significado diferente cuando se acompaña de una sonrisa o un ceño fruncido... Una segunda función es que las claves no verbales podrían intensificar o reducir la expresión de emoción. Simples palabras podrían no ser capaces de llevar toda la información emocional que alguien quiera transmitir (traducción propia) ${ }^{12}$ (p.776).

$11 \quad$ A message contains not only a single meaning but consists of four distinct communicative layers. The first layer consists of the factual content of the message, i.e., about what one wants to inform one's counterpart. The second layer, self-revelation, refers to what one discloses about oneself with the message. The third layer provides information about the relationship between the communicators, i.e., what one thinks about the other and how he or she relates to him or her. The fourth layer, appeal, indicates to what one wants to induce one's counterpart (Griessmair y Koeszegi, 2009: 216-217).

12 Non-verbal displays have several social functions. One function is that the ambiguity of the intended emotion expression is reduced. For example, a message may have a different meaning when accompanied by a smile or a frown ... A second function is that non-verbal cues may intensify or tone down the emotion expression (Lee $y$ Wagner, 2002). Mere words may not be able to carry all the emotional information that someone wants to convey (Derks; Fischer y Bos, 2008: 776). 
La expresión no verbal de emociones $y$ actitudes por medio de gestos $y$ de cambios en la entonación, proveen claves a los interlocutores sobre la intención del otro, la veracidad del mensaje e incluso la personalidad y la historia del individuo, elementos que vienen a formar parte de la base conceptual común entre los interlocutores y refuerzan la cooperación. De forma similar, Carter comenta sobre la posición de Ekman ante las claves no verbales:

Algunas de las expresiones faciales de emoción - al menos aquellas que indican felicidad, miedo, enojo, asco, tristeza, sufrimiento, entre otras - son universales, las mismas para todo el mundo independientemente de la edad, el sexo, la raza, o la cultura. La contribución del lenguaje corporal es tan fundamental que dependemos fuertemente de ella para comprendernos los unos a los otros (traducción propia) ${ }^{13}$ (2003: 32).

Todas estas funciones de la comunicación no verbal, sostienen algunas y algunos investigadores, se encuentran ausentes en la CMC, por lo tanto, el proceso de reconocimiento del otro como individuo no podría darse de la misma forma en un ambiente despersonalizado que cara a cara.

En "Emoticons and social interaction on the internet: the importance of social context", Derks; Bos y Grumbkow (2007), mencionan como una de las principales limitaciones que se le achacan a la $\mathrm{CMC}$, la falta de espontaneidad, al ser necesario escribir la información, lo cual retrasa la interacción y "la formación de impresiones" y crea un tiempo de respuesta en el que los individuos pueden pensar sus reacciones con más calma, lo que altera la veracidad de la reacción y la hace más impersonal ( $\mathrm{p}$. 843-844). Como resultado de su investigación, concluyen que:

$13 \quad$ Some of the facial expressions of emotion - at least those indicating happiness, fear, anger, disgust, sadness, distress, and others - are universal, the same for all people regardless of age, sex, race, or culture. So fundamental is the contribution of body language that we rely heavily on it to understand each other (Carter, 2003: 32).
Por la falta de claves no verbales en la CMC, se requiere de más mensajes y por ende, de más tiempo, para incrementar el aspecto relacional de la comunicación, al mismo nivel de la comunicación cara a cara y como resultado se obtienen más problemas de comunicación (traducción propia) ${ }^{14}$ (Derks; Bos y Grumbkow, 2007: 848).

De forma similar, Carter (2003) establece que "ninguna comunicación transaccional ocurre durante la CMC. Transmisor y receptor carecen de la habilidad para transmitir mensajes simultáneamente, la cual provee la base para una comunicación interpersonal congruente" (traducción propia) ${ }^{15}$ (p. 34). Por otra parte, Lo (2008) propone que el uso de claves cuasino verbales -en este caso los emoticones- sí ayudan a transmitir emociones $y$ actitudes en la comunicación; sin embargo, no dejan de ser formas verbales al ser expresadas en forma gráfica (p. 595). La "anonimidad visual" y la necesidad de "texto" en la CMC, son la base, según Joinson (2001), de la creencia que "La CMC carecerá de la riqueza de la interacción cara a cara normal y por ende, será orientada a las tareas y tendrá un bajo contenido socio-emocional... [y] desalentaría la comprensión de los otros" (traducción propia) ${ }^{16}$ (p. 177). Ahora bien, a pesar de las limitaciones que presenta la CMC, hay investigaciones que demuestran la utilidad de claves "cuasi-no verbales", quizá no para

14 Because of the lack of nonverbal cues in $C M C$ there are more messages required, and thus more time, to raise the relational aspects of communication to the same level as in face-to-face communication (Walther y D'Addario, 2001) and as a result there are more miscommunications (Erkens; Theil; Kanselaar; Prangsma y Jaspers, 2002) (Derks; Bos y Grumbkow, 2007: 848).

15 No transactional communication comes into play during CMC. Sender and receiver lack the ability to transmit messages simultaneously, which provides the basis for congruent interpersonal communication (Carter, 2003: 34).

$16 \quad$ CMC will lack the richness of normal face-to-face interaction and hence be task oriented and low in socio-emotional content... [and it] would discourage awareness of others (Joinson, 2001:177). 
substituir por completo a las claves no verbales, sino para solventar en parte su falta y así lograr una interacción mucho más efectiva en medios textuales.

\section{LAS CLAVES CUASI-NO VERBALES EN LA CMC}

Se analizaran ahora algunas de las formas cuasi-no verbales que encontramos en la CMC. Como primer ejemplo, tenemos los emoticones. Los emoticones, en su forma más básica, son figuras formadas con elementos tipográficos que representan una facción o emoción. Estos son quizá la forma de claves cuasi-no verbales más utilizada en la mayoría de medios de CMC: mensajes de texto, chatrooms, juegos de video en línea, etc. Según Emoticons \& Smiley Page, entre los emoticones básicos se encuentran:

:-) Smiley estándar [estas bromeando; satisfecho].

:) Smiley estándar [para personas perezosas].
,-) Smiley guiñando el ojo [no es su intención, aun cuando esté bromeando].

;-) Smiley guiñando el ojo [ver anterior].

:-> [Acompaña un comentario realmente sarcástico].

(-: [Smiley para zurdos].

:-( Smiley triste [no está bromeando; no está satisfecho].

$:<$ Smiley muy triste.

:C Smiley muy triste.

:-* Smiley dando un beso.

Nota: La traducción de los emoticones fue realizada por el autor.

Estos símbolos ayudan al emisor, no solo porque le añade el aspecto emocional al mensaje, sino también porque le evita malos entendidos cuando el mensaje escrito es ambiguo. Además de los emoticones tipográficos, los servicios de mensajería instantánea han desarrollado emoticones gráficos y animados, los cuales proveen un mensaje más claro, al presentar una imagen gráfica en movimiento realizando una expresión facial dada, algunas veces acompañada también de sonido. Algunos ejemplos son:

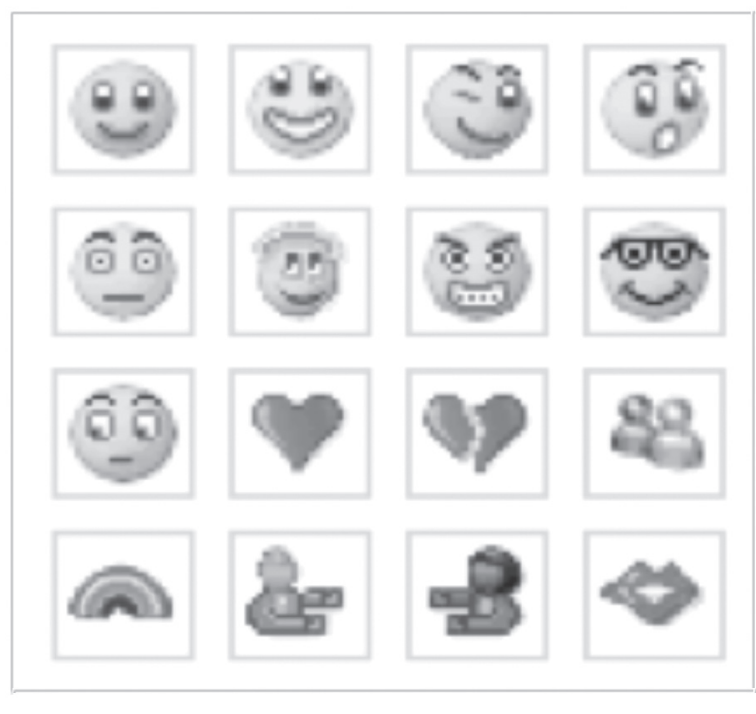

FIGURA 1

EMOTICONES DE MESSENGERADICTOS

En "A stylistic analysis of graphic emoticons: can they be candidates for a universal visual language of the future?", Junichi Azuma et ál. (2008) analizan el uso de emoticones como formas casi universales de comunicación que asemejan a las claves no verbales y que su fácil interpretación por hablantes de distintas lenguas sugiere que actúan como "Elementos 
léxicos de un lenguaje primitivo universal visualmente construido" (traducción propia) ${ }^{17}$, refiriéndose a las claves no verbales "naturales" y "universales", proponen que:

Es natural que los emoticones o smilies hayan ingresado gradualmente en el ciberespacio para proveerle a la comunicación exclusivamente, de lenguaje $y$ en apariencia exclusivamente, lógica del ciberespacio un toque emocional $y$ humano ;-). De alguna manera, un emoticón, usualmente colocado al final de una frase u oración, es una versión tipográfica de una característica paralingüística o prosódica (traducción propia) ${ }^{18}$.

El uso de emoticones viene a sustituir la expresión facial a la que se recurriría en la interacción cara a cara en un medio eminentemente textual y despersonalizado. En la misma línea, Derks; Bos y Grumbkow (citando a Thompson y Foulger, 1996: 843) mencionan que:

Ya que los emoticones podrían servir como substitutos no verbales, al sugerir expresiones faciales, podrían añadirle un componente paralingüístico al mensaje. Los emoticones podrían entonces realzar el intercambio de información social al proveer claves sociales adicionales más allá de lo que se encuentra en el texto de un mensaje (traducción propia) ${ }^{19}$ (2007:

Lexical items of a primitive universal visually constructed language (Junichi Azuma et ál, 2008).

It is natural that emoticons or smilies have gradually entered cyberspace to provide the language-only and seemingly logic-only cyberspace communication with an emotional and human touch ;-). In a sense, an emoticon, very often placed at the end of a phrase or a sentence, is a typographic version of a paralinguistic or prosodic feature (Junichi Azuma et ál., 2008).

19 Since emoticons may serve as nonverbal surrogates, suggestive of facial expression, they may add a paralinguistic component to a message. Emoticons may thus enhance the exchange of social information by providing additional social cues beyond what is found in the text of a message (Derks; Bos y Grumbkow, 2007: 843).
843) [y concluyen, resaltando siempre las limitaciones del medio, que el contexto social es de gran importancia en la CMC $y$ que el uso de emoticones refleja esta necesidad en los usuarios] (p. 847).

Por su parte Lo (2008) expone las conclusiones de su estudio, basado en un experimento realizado con 137 usuarios de un servicio de mensajería instantánea y menciona:

Los resultados indican que cuando los usuarios de internet se enfrentan solo a un texto sin emoticones, la mayoría de las personas no puede percibir correctamente la intención de la emoción, la actitud o la atención. Sin embargo, cuando se incluyen emoticones en el mismo contexto, la percepción del mensaje por parte del receptor comienza a cambiar significativamente. También, cuando se utilizan emoticones con un significado opuesto, el receptor muestra una extrema diferencia en la percepción. Los emoticones le permiten a los receptores comprender correctamente el nivel y dirección de la expresión de la emoción, la actitud y la atención. Estos resultados prueban que los emoticones realizan funciones de comunicación no verbal. Por ende, este estudio proclama audazmente que los emoticones utilizados en la comunicación en línea podrían llamarse claves cuasi no verbales (traducción propia) ${ }^{20}$ (p.597).

20 Results show that when Internet users are faced with pure text without emoticons, most people cannot perceive the correct emotion, attitude, and attention intents. However, when emoticons are added in the same context, the receiver's perception of the messages starts to significantly change. Also, when opposite-meaning emoticons are used, the receiver shows extreme difference in perceptions. Emoticons allow receivers to correctly understand the level and direction of emotion, attitude, and attention expression. These results prove that emoticons perform nonverbal communication functions. Therefore, this study boldly proclaims that emoticons used in online communication may also be called quasi-nonverbal cues (Lo, 2008:597). 
Los resultados del experimento de Lo (2008) dejan en claro la funcionalidad de los emoticones como substitutos de las claves no verbales de la comunicación cara a cara. De igual forma, Derks, Fischer y Bos (2008) exponen como resultado de su investigación, que "los emoticones abundan en la CMC y no hay indicio de que la смc sea un medio impersonal, ni tampoco que sea más difícil comunicar emociones en línea" (traducción propia) ${ }^{21}$ (p. 779). Por ejemplo, en un mensaje como "mañana tengo examen y no he estudiado", al incluir un emoticón como :( le damos un contexto diferente a si utilizamos :D. En el primer caso se indica tristeza ante la situación, mientras que en el segundo caso se denotaría una actitud despreocupada $y$ hasta jocosa ante la potencial desgracia. En otro ejemplo, si el mensaje dice "no me gusta su actitud", :D indica que estamos bromeando, mientras que ]:[ indica que estamos molestos y que el mensaje debe entenderse textualmente. Como se ve, el uso de emoticones no solo ayuda a evitar, en la manera de lo posible, las malas interpretaciones de los mensajes, sino que también expresan nuestras emociones $y$ actitudes para con los "otros" de una forma más "natural" en medios textuales eminentemente artificiales, pero altamente sociales.

Además del uso de emoticones para expresar emociones $y$ actitudes en la CMC, los usuarios de medios electrónicos de comunicación utilizan otras formas de expresión que asemejan a claves no verbales, como lo son algunas claves tipográficas y los emotes en los MMORPG y a pesar de que a diferencia de los emoticones, que son íconos que representan claramente un gesto o expresión facial, estas claves textuales necesitan ser "leídas" como texto escrito, dichas claves cumplen efectivamente la misma función de expresión emocional en sustitución de su contraparte no verbal, de la comunicación cara a cara. Griessmair y Koeszegi (2009) incluyen entre lo que llaman un "paralenguaje electrónico" el uso de acrónimos y de abreviaciones para

21 Emotions are abundant in $C M C$, and there is no indication that CMC is an impersonal medium, nor that it is more difficult to communicate emotions online (Derks; Fischer y Bos, 2008: 779). transmitir información "socio-emocional" (p. 215). Varnhagen et ál. (2009) mencionan, de manera similar, formas alternativas para expresar emociones: "Encontramos un número de claves a emociones, que van desde el uso de palabras de emoción (e.g. jajaja) y acrónimos (e.g. lol) hasta el uso excesivo de puntuación (e.g. !!!!) y mayúsculas (e.g. QUÉ) para expresar una emoción" (traducción propia) ${ }^{22}$. Similares a los emoticones, estas claves tipográficas, ya sea al final de un mensaje textual o por sí solas, contribuyen a construir un mensaje más claro, al incluir emociones y actitudes al mensaje escrito. Volviendo al ejemplo anterior de "mañana tengo examen y no he estudiado", podemos substituir el emoticón :D por la palabra de emoción "jajaja" o por el acrónimo "lol" (por las iniciales en inglés ${ }^{23}$ de "riéndose a carcajadas' (traducción propia)). Si queremos darle una connotación jocosa a la situación, o por la repetición de signos de admiración "!!!!" para indicar desesperación. Por su parte, el uso de mayúsculas sustituye a los cambios en la entonación en el lenguaje oral para indicar un cambio de emoción. Por ejemplo, en un contexto determinado, la connotación y el impacto del mensaje "déjeme en paz" cambia totalmente si lo escribimos en mayúscula; "DÉJEME EN PAZ", lo cual indicaría un incremento en el tono de voz que denota impaciencia o ira, de igual forma que un mensaje como "MAÑANA ME VOY DE VIAJE" podría indicar entusiasmo.

Por otro lado, tenemos los "comandos gesticulares" o emotes, muy utilizados en algunos MMORPG y que llevan las claves cuasino verbales a un nivel mucho más elevado de comunicación. Tal y como lo exponen Innocent $y$ Haines, en "Nonverbal Communication in Multiplayer Game Worlds":

Debido a la naturaleza social de la mayoría de mundos virtuales, la comunicación jugador a jugador podría considerarse

22 We found a number of cues to emotion, ranging from use of emotion words (e.g., hahaha) and acronyms (e.g., lol) to ecessive use of punctuation (e.g., !!!!!) and upper case (e.g., WHAT) to express emotion (Varnhagen et ál, 2009). 
tan importante como los sistemas (la mecánica, lo administrativo, lo técnico) usados para gobernar y mantener el persistente mundo, ya que esta comunicación le da forma a la identidad de los jugadores $y$ a la personalidad del mismo mundo (traducción propia) ${ }^{24}(2007)$.

Para brindarles a los y las jugadoras la posibilidad de comunicarse e identificarse, los videojuegos en línea cuentan con un chat básico, además de un personaje o avatar que representa a los y las jugadoras en el mundo virtual. En este chat, los y las jugadoras recurren a las mismas claves cuasi-no verbales que se mencionaron anteriormente -emoticones y claves tipográficas-, pero se incluye en algunos casos la posibilidad de utilizar emotes, que llevan la comunicación no verbal a otro nivel. Por "comandos gesticulares" o emotes, se entienden comandos utilizados en MMORPGs para que nuestro personaje demuestre una emoción ya sea gesticulando, desplegando una oración que refleja dicho sentimiento o ambas. Se analizará este tipo de comunicación cuasi-no verbal en el videojuego The World of Warcraft (2007), uno de los MMORPGs más populares y que cuenta con 12 millones de usuarios en todo el mundo en el servidor oficial de Blizzard Entertainment (2010), sin contar los jugadores en los distintos servidores privados gratuitos.

The World of Warcraft (WoW) es un MMORPG con un alto grado de socialización

24 Due to the social nature of most virtual worlds, player-to-player communication may be seen as equally important as the systems (game mechanics, administrative, technical) used to govern and maintain the persistent world as this communication shapes the players identity and the character of the world itself (Innocent $y$ Haines, 2007). entre usuarios. Para que un(a) jugador(a) logre explotar la experiencia de juego al máximo, requiere de la ayuda de otros usuarios. WoW no solo demanda del jugador o jugadora llevar a cabo misiones individuales, sino también raids - juegos grupales que requieren de entre 5, $10 \mathrm{o}$ hasta 25 jugadores dependiendo del nivel de dificultad-y los battlegrounds - juegos en modo jugador versus jugador - en los que grupos de las dos facciones enemigas (Aliance y Horde) se enfrentan en un campo de batalla para adquirir honor y reputación. Además de las misiones, los raids y los battlegrounds, los y las jugadoras de WoW se unen en guilds, que son cofradías de jugadores y jugadoras que ponen sus propias reglas para aceptar a nuevos miembros y que les otorgan ciertos beneficios, tales como facilidad de organizar raids, conseguir ayuda para hacer misiones complejas, conseguir equipo, armas $y$ materiales, entre otros. Esto demanda de los $y$ las jugadoras una buena reputación para ser aceptados en algún guild. Los y las jugadoras también aprenden profesiones para las cuales necesitan de materiales $y$ productos que otros usuarios fabrican u obtienen. El o la jugadora de WoW realiza transacciones, vende productos o incluso, compra o canjea materiales con otros usuarios tanto para obtener oro (la moneda utilizada en el juego) como para ganar experiencia en dicha profesión. Todas estas actividades requieren de la interacción social entre jugadores y demandan una comunicación efectiva en la medida de lo posible.

Como medio de comunicación dentro del juego, WoW posee una ventana de chat en la que se accede a las diferentes formas de comunicación, ya sea texto escrito, emoticones tipográficos o emotes, las cuales se despliegan tanto en el mismo chat como sobre el personaje en forma de un globo de diálogo (ver figuras 2 y 3 ). 
FIGURAS 2

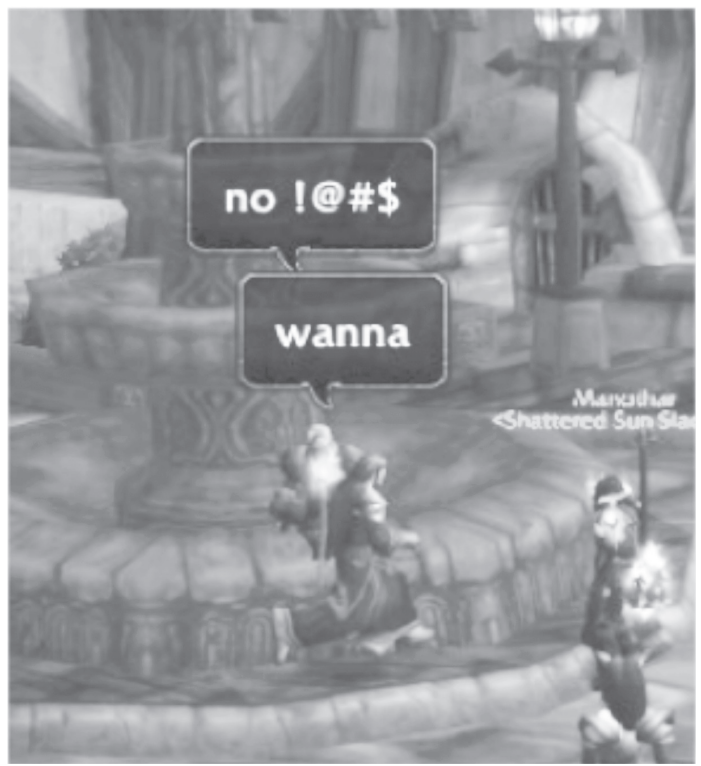

FIGURA 3

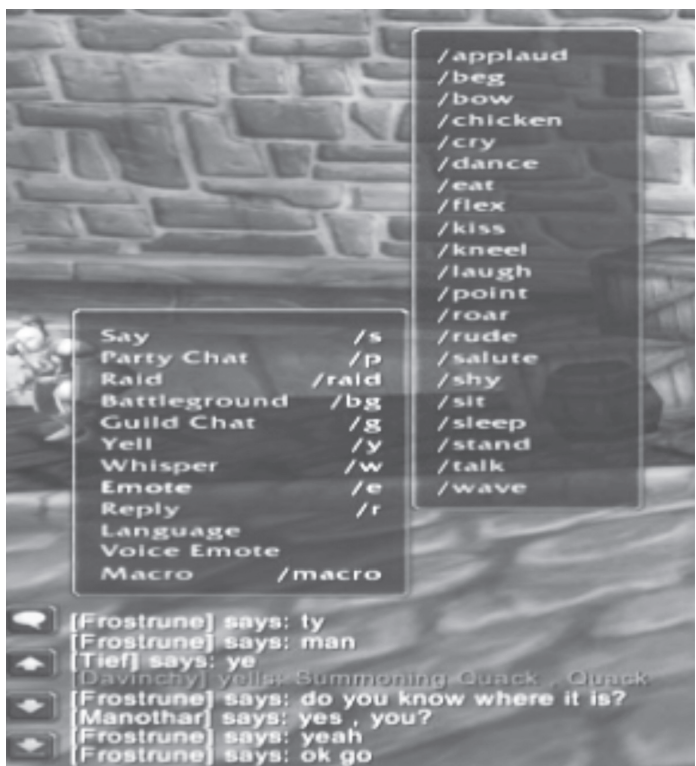

Fuente: capturas de pantallas de chat de WoW.

El o la jugadora tiene toda una gama de opciones de emotes para utilizar en una conversación con otros jugadores, que van desde emotes que despliegan un texto explicativo y gesto del personaje, hasta emotes de voz, en los que el personaje reproduce oralmente alguna frase u oración predeterminada de acuerdo al comando que se utiliza.

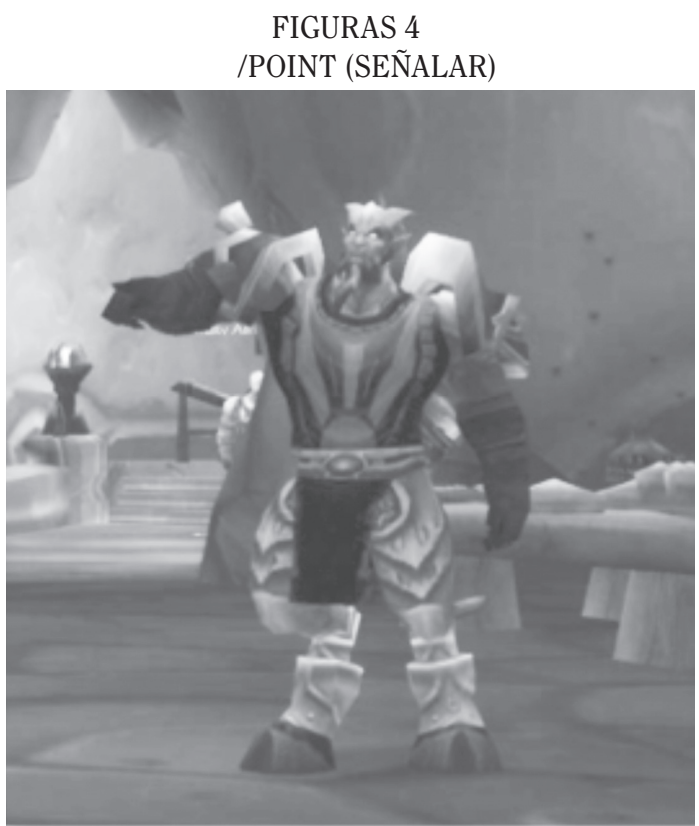

Estos últimos, sin embargo, los dejaremos de lado ya que lo que interesa, para efectos del presente estudio, son las claves "cuasi-no verbales". Entre los emotes básicos con que cuenta el chat de WoW encontramos los siguientes:

FIGURA 5

/CRY (LLORAR)

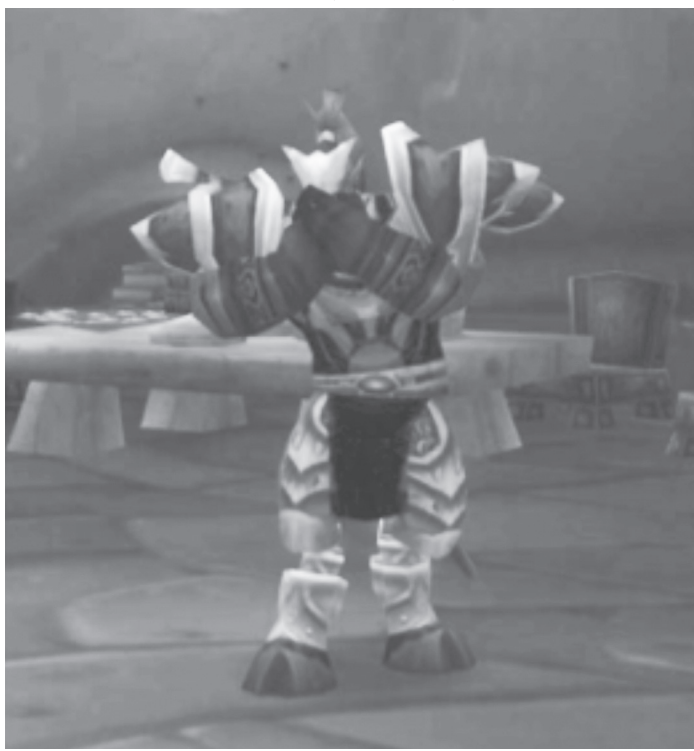


FIGURA 6 /SHY (TÍMIDO)

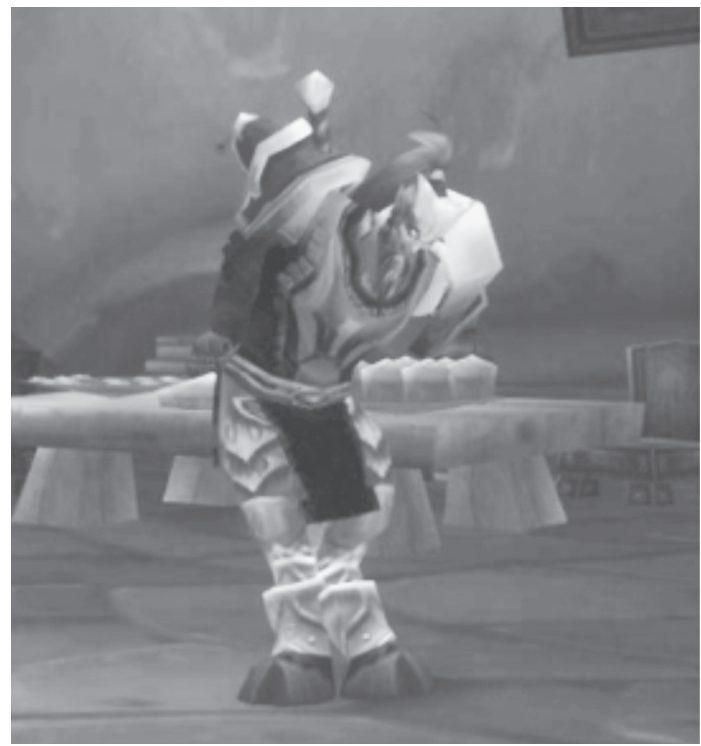

FIGURA 7 /SALUTE (SALUDAR)

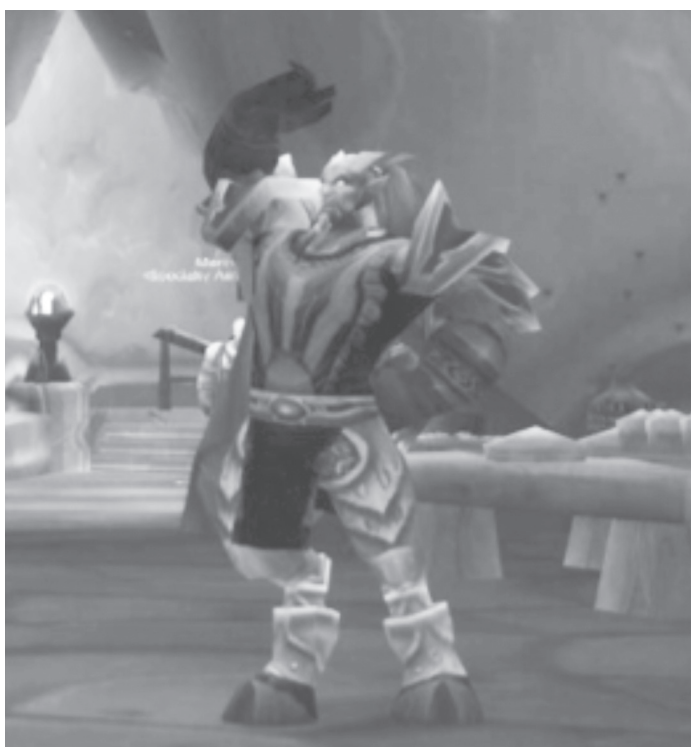

Fuente: capturas de pantalla de emotes en WoW

Como lo muestran las imágenes anteriores, cada uno de estos comandos va acompañado de un gesto del personaje, como lenguaje corporal similar al de la función que cumplen los emoticones animados, que representa la intención del emisor, agregándole un toque mucho más personal que un simple "Hola", "Estoy triste" o "Me da pena", por ejemplo. Además de estos emotes animados, hay otros comandos que simplemente despliegan un texto en el chat indicando la intensión del emisor. Cuando se dirige /slap (abofetear) a un jugador, se despliega el texto "[Jugador x] slaps you across the face. Ouch!" ([Jugador x] te abofetea en la cara. Ay!) al igual que /disappointed (decepcionado) indicará "[Jugador x] frowns with disappointment at you" ([Jugador $\mathrm{x}] \mathrm{le}$ frunce el ceño decepcionado). En estos casos, a pesar de que la acción aparece como texto escrito, alude a una acción o expresión corporal que indica un sentimiento o actitud específica. Además, no es el jugador el que escribe el texto completo sino el programa el que interpreta el comando y lo presenta de esta forma (ver figura 8 , segunda línea del chat).
FIGURA 8

CHAT DE WOW-CACHETADA

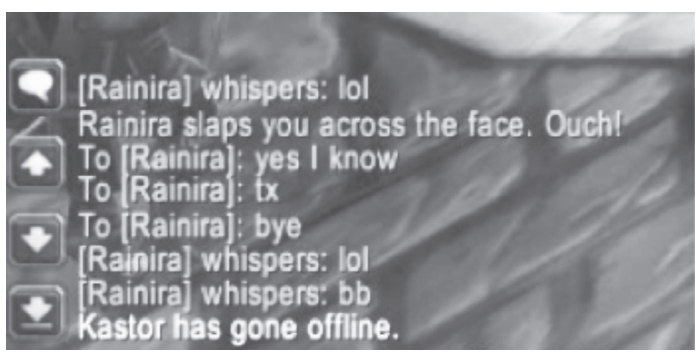

Fuente: Captura de pantalla de WoW.

Estas funciones les permiten a las y los jugadores de WoW llevar la experiencia de socialización en el mundo virtual a un nivel más personal, utilizando expresiones más allá del texto escrito despersonalizado y agregándole el elemento emocional que revela parte de la actitud $y$ de la personalidad del usuario. Los usuarios de MMORPGs, como en el caso de WoW, deben lidiar con el distanciamiento característico de la CMC, con la incertidumbre que genera la comunicación entre sujetos ocultos tras la imagen de un personaje ficticio en un mundo 
ficticio y al uso de un idioma que no necesariamente dominan, dado que la mayoría de servidores de WoW utilizan un idioma específico -muchas veces el inglés - como lenguaje oficial, aumentando así las posibilidades de malas interpretaciones. Estas faltas características de los videojuegos en línea, realzan la necesidad de este tipo de claves no verbales para darse a entender apropiadamente y aunque quizá más inconscientemente, revelarse como individuos y crear una reputación en el medio. De esta reputación y de la forma en que los usuarios se revelen ante los demás, puede depender que un o una jugadora sea invitada a participar en ciertos eventos grupales o que sea rechazada.

\section{CONCLUSIONES}

Se ha visto como la CMC no es un espacio de comunicación totalmente estéril en términos de socialización, sino que, a pesar de sus limitaciones en proximidad $y$ visión, ha desarrollado características muy similares a la comunicación cara a cara, permitiéndoles a los y las usuarias revelar aspectos de sí mismos como individuos en espacios sociales virtuales. Se ve como las claves "cuasi-no verbales" utilizadas en la CMC reproducen elementos kinésicos y paralingüísticos de la comunicación cara a cara que se creían ausentes $y$ por ende, limitantes de la posibilidad de una individuación como la plantea Habermas (1990). Es un hecho que muchas personas utilizan espacios virtuales para socializar, en los que se crean relaciones amistosas, o incluso amorosas, que no serían posibles sin un grado de revelación del individuo ante el otro. Los emoticones, las claves tipográficas y los emotes, aunque presuponen un nivel de incertidumbre ante la posibilidad de una construcción falsa del individuo, son utilizadas cada vez más para reducir el vacío que dejan las claves no verbales de la comunicación cara a cara y se apoyan en el sentido de cooperación mutua que, según Tomasello (2008), es el aspecto que marcó el desarrollo evolutivo de la comunicación humana. Sería muy arriesgado asumir que las claves "cuasi-no verbales" actualmente logran sustituir la efectividad de la comunicación cara a cara -que ya de por sí, no es $100 \%$ efectiva y es también propensa a malas interpretaciones y mani- pulaciones por parte de los interlocutores - sin embargo, sí podemos asegurar que estas claves generan nuevas posibilidades de comunicación mucho más eficaces que un simple texto escrito, denotando la necesidad del ser humano de buscar formas de posicionarse como individuo ante la sociedad sin importar el medio de comunicación que se utilice.

\section{BIBLIOGRAFÍA}

Azuma, Junichi et ál. "A stylistic analysis of graphic emoticons: can they be candidates for a universal visual language of the future?". Proceeding of World Conference on Educational Media, Hypermedia and Telecommunications. Graz University of Technology, 2008: 972-977.

Blizzard Entertainment. World of Warcraft: the burning crusade. California, 2007.

Blizzard Entertainment Company. "World of Warcraft Subscriber Base Reaches 12 Million Worldwide”. Blizzard Entertainment Press Releases. 2010. En: $<$ http://us.blizzard.com/en-us/company/ press/pressreleases.html? $101007>$ [consultado el 21 de marzo de 2011].

Carter, Kimberly A. "Type me how you feel: quasi-nonverbal cues in computermediated communication". ETC: A Review of General Semantics 60 (1). 2003.

Derks, Daantje; Fischer, Agneta H. y Bos, Arjan E.R. "The role of emotion in computermediated communication: a review". Computers in Human Behavior 24. 2008: 766-785.

Derks, Daantje; Bos, Arjan E.R. y von Grumbkow, Jasper. "Emoticons and social interaction on the Internet: the importance of social context". Computers in Human Behavior 23. 2007: 842-849.

Duke, Charles R. "Nonverbal behavior and the communication process". College composition and communication 25 (5). 1974: 397-404.

Emoticons \& Smile Page. Muller-godschalk. En: <http://www.muller-godschalk.com/ 
emoticon.html> [consultado el 20 de noviembre de 2009].

Griessmair, Michele y Koeszegi, Sabine. "Exploring the cognitive-emotional fugue in electronic negotiations". Group Decision \& Negotiation 18(3). 2009: 213-234.

Habermas, Jürgen. "Individuación por vía de socialización. Sobre la teoría de la subjetividad de George Herbert Mead". Pensamiento Postmetafísico. España. Taurus, 1990: 188-239.

Hein, A.; Step, M. y Turkstra, L. "Show me what you mean: nonverbal communication theory and application". The ASHA Leader. American Speech-LanguageHearing Association, 2003.

Innocent, Troy y Haines, Stewart. "Nonverbal communication in Multiplayer Game Worlds". ACM International Conference Proceeding Series 305. 2007.

Joinson, Adam N. "Self-disclosure in computermediated communication: the role of self-awareness and visual anonymity". European Journal of Social Psychology 31(2). 2001: 177-192.

MessengerAdictos. "Emoticonos". En: <www. messengeradictos.com/emoticonos> [consultado el 20 de noviembre de 2009].

Lo, Shao-Kang. "The nonverbal communication functions of emoticons in computer-mediated communication". CyberPsychology \& Behavior 11(5). 2008: 595-597.

Tomasello, Michael. Origins of the human communication. Cambridge: MIT Press, 2008.

Varnhagen, Connie K. et ál. "lol: new language and spelling in instant messaging". Reading and Writing. 2009.

Fecha de ingreso: 19/05/2010

Fecha de aprobación: 19/07/2011 